\title{
Implementation of stable solutions in a restricted matching market ${ }^{\star}$
}

\author{
Antonio Romero-Medina \\ Departament of Economics, University of California, Riverside, CA 92521, USA \\ (e-mail: aromero@ucrac1.ucr.edu)
}

\begin{abstract}
I analyze the admission mechanism used in Spanish universities. The system is open to strategic manipulation. This is because students are not allowed to express the whole list of available options. However, the mechanism implements the set of stable matchings in Nash equilibrium and the student's optimum in strong equilibrium. The mechanism also implements the students' optimum, in Nash equilibrium, under the class of "non-reverse" preferences. All these properties come from the fact that colleges do not have the opportunity to misrepresent their preferences.
\end{abstract}

Key words: Matching markets, implementation

JEL classification: C71, C78, D78, J24

\section{Introduction}

The admission of students to universities is an important economic decision. It affects students' lives in many ways and mobilizes many public resources. These resources admit other uses even within the global higher education budget.

The process that matches students and universities resembles a market with some peculiar features. Demand and supply of positions are discrete variables. Prices (fees) are not flexible and cannot play the usual role of adjusting supply and demand. Yet, efficiency considerations make it important to take the preferences of students into account, and to adjust the supply of positions to social demand.

\footnotetext{
* This paper is based on the second chapter of my Ph.D. dissertation submitted to the Universitat Autonoma de Barcelona. I wish to thank my advisor, Professor Salvador Barberá, for his efforts in supervision and very useful suggestions. I am grateful to José Alcalde, David Perez-Castrillo, Jordi Massó, Antonio Cabrales, Jorge Nieto, and an anonymous referee for their helpful comments. Financial support from the Generalitat de Catalunya, Instituto de Estudios Fiscales and DGCYT under project PB 92-0590, is gratefully acknowleged.
} 
For all these reasons, I want to study a model capturing the essential features of the process whereby a public office might intermediate among students who seek a position in the higher educational system and the suppliers of these positions - the universities. The appropriate model is well known: it is called a many-to-one two-sided matching problem. This model has been extensively studied since the seminal work of Gale and Shapley [3] (for an excellent survey, see Roth and Sotomayor [9]). I have chosen the details of the model so that they fit the institutional features of the system which is presently used in Spain to decide on which students should be admitted to each available college position. The Spanish model limits the number of options that students can declare and gives no chance to the colleges to act strategically. This analysis can be applied to many other cases, for instance the allocation of interns to hospitals in the same country, and can be adapted to cover similar procedures used in other countries.

This paper is concentrated on the strategic aspect of such an allocation process. The system used in Spain is open to manipulation because students are not allowed to express the whole list of their desired options. In the short run these manipulations do not necessarily disrupt the stability of the allocation. Technically speaking, I prove that stable allocations will be implemented under different equilibrium concepts. But I also argue that strategic behavior may result in misperceptions of the true preferences of the students, and eventually distort the long run allocation of funds within the educational system.

After a few definitions Sect. 2 studies the specific features of the Spanish system. Section 3 analyzes their strategic and allocational consequences. Section 4 contains the main results and their implications for the long-run allocation of resources. Short conclusions follow in Sect. 5.

\section{The model}

In a bilateral market there are two finite disjoint sets. Let $S=\left\{s_{1}, s_{2}, \ldots, s_{n}\right\}$ be the set of students and let $C=\left\{c_{1}, c_{2}, \ldots c_{m}\right\}$ be the set of colleges. Each $s_{i}$ is endowed with a complete, transitive preference relation $P\left(s_{i}\right)$ on $C \cup\left\{\mathrm{s}_{i}\right\}{ }^{1}$. For $c_{j}, c_{k} \in C, c_{j} P\left(s_{i}\right) c_{k}$ means that $s_{i}$ prefers to be matched to college $c_{j}$ rather than $c_{k} ; s_{i} P\left(s_{i}\right) c_{j}$ means that $s_{i}$ prefers to be unmatched rather than be matched with $c_{j}$. Similarly, each college $c_{j}$ is endowed with a complete transitive preference relation $P\left(c_{j}\right)$ on $S \cup\left\{c_{j}\right\}$.

I denote preference profiles by $P$. The preferences of any agent are going to be represented by a list, $P\left(s_{i}\right)=c_{1}, c_{2}, \ldots, s_{i}, c_{l}, c_{l+1}, \ldots, c_{m}$. In this list, $c_{1}$ is $s_{i}$ 's most preferred college, $c_{2}$ is his second more preferred college, and so on. $s_{i}$ represents the position where he places the possibility of not being matched. Any college after this position is unacceptable for $s_{i}$.

Every college $c_{j}$ has a maximum number of available admissions $k_{j}$. This quota is determined exogenously and reflects the physical capacity of each college.

A matching is a correspondence $G: C \cup S \Rightarrow C \cup S$, such that:

1. $\left|G\left(s_{i}\right)\right|=1$, for every student $s_{i} \in S$, and $G\left(s_{i}\right)=s_{i}$ if $G\left(s_{i}\right) \notin C$. 
2. $\left|G\left(c_{j}\right)\right|=k_{j}$, for every college $c_{j}$. If the cardinality of $G\left(c_{j}\right)$, say $r$, is smaller than $k_{j}$, then $G\left(c_{j}\right)$ has $k_{j}-r$ copies of $c_{j}$ representing empty allocations.

3. $G\left(s_{i}\right)=c_{j}$ if and only if $s_{i} \in G\left(c_{j}\right)$.

I denote $G\left(s_{i}\right)$ by $G_{i}$ and $G\left(c_{j}\right)$ by $G_{j}$. Subindexes $i$ and $j$ refer to students and colleges, respectively. The set $\mathscr{C}$ represents all possible matchings between students and colleges.

As the students have only one college in each stable matching, their preferences over matchings correspond to their preferences over individual agents. The same cannot be said about colleges: every college having a quota higher than one should be able to compare groups of students.

Definition 1 A preference relation $P_{j}^{\diamond}$ for $c_{j} \in C$ over the set of students is responsive with respect to the preferences $P_{j}$ over $S \cup\left\{c_{j}\right\}$ if for every $G_{j}{ }^{\prime}=$ $G_{j} \cup\left\{s_{k}\right\} \backslash\{\sigma\}$, with $\sigma \in G_{j}$ and $s_{k} \notin G_{j}$, $c_{j}$ prefers $G_{j}^{\prime}$ (under $P_{j}^{\diamond}$ ) over $G_{j}$ if an only if $c_{j}$ prefers $s_{k}$ to $\sigma$ (under $P_{j}$ ).

I will assume that preferences of colleges over groups of students are responsive to their preferences over individual students.

A market is described by a triplet $(C, S, P)$, where $C$ is a set of colleges, $S$ is a set of students and $P$ stands for the preferences of both sides. I will use this notation to provide a brief description of basic concepts.

Definition 2 Let $G$ be a matching in the market $(C, S, P)$. $G$ is individually rational if it satisfies:

i) for all $s_{i} \in S$, $\left[G_{i} \in C\right] \rightarrow\left[G_{i} P_{i} s_{i}\right]$, and

ii) for all $c_{j} \in C$, and for all $s_{i} \in S,\left[s_{i} \in G_{j}\right] \rightarrow\left[s_{i} P_{j} c_{j}\right]$.

Definition 3 Let $G$ be a matching in the market $(C, S, P)$. The pair $\left(c_{j}, s_{i}\right)$ blocks $G$ if:

i) $\left[G_{j} \cup\left\{s_{i}\right\} \backslash\{\sigma\}\right] P_{j} G_{j}$, and

ii) $c_{j} P_{i} G_{i}$.

Definition 4 A matching $G$ is stable for $(C, S, P)$ if it is individually rational and it is not blocked by any pair $\left(c_{j}, s_{i}\right)$.

I will denote the set of stable matchings of a market $(C, S, P)$ as $\mathscr{E}$. Given a market $(C, S, P), \mathscr{E}\left(s_{i}\right)$ stands for the set of elements in $C$ assigned to $s_{i}$ in some stable matching; $e\left(s_{i}\right)$ is an element of $\mathscr{E}\left(s_{i}\right)$. The existence of at least one stable matching for each market was originally established by Gale and Shapley [3].

\subsection{The Spanish system}

In Spain colleges admit students by using a ranking based on the students' previous studies, their grades in high school, and grades from an admittance examination. This priority criterion leaves no room for colleges to influence their 
admission choices. Students who have already finished high school and want to go to college must pass an exam. After that they submit an ordered list containing a limited number, $t$, of possible declarations. This number is common to all students. Each university offers positions for different types of training, for example, physics, mathematics, history, economics, etc. Students can simultaneously apply for one position in each one of the different schools of each university. To keep with the traditional terminology in the matching literature, I refer to each one of these schools as a separate college.

Once the central allocation office has received all the lists, the Deferred Acceptance Procedure (D.A.P.) is used to allocate students to colleges. First, students are arbitrarily ordered. The first student is assigned to the most preferred college for which this student is acceptable. At each stage the corresponding student is assigned tentatively to his most preferred college. However, if the college has already completed its quota with other more preferred students, he is refused, and tentatively matched with the following college in the student's ranking. The algorithm continues until each student is assigned to a college or there is no college left in their list. It is only then that the tentative match becomes final. This algorithm leads to the stable matching that all students unanimously consider best for them.

Formally the problem is described by four elements $(C, S, P, t)$, already defined, where the number of possible declarations of the students, and the colleges preferences are fixed exogenously. Therefore, the declarations of the agents and the results of the mechanism will only be determined by the students' preferences. I need to distinguish between the statements that agents would make about their preferred colleges if unrestricted, and those that they will make when constrained to declare only a fixed number $t$ of college positions.

I denote by $q_{i}$ the lists of colleges declared by agent $s_{i}$ from better to worse, regardless of whether they correspond to $s_{i}$ 's sincere preferences or not; $q_{i}^{t}$ represents a student's list when $s_{i}$ is limited to declare at most $t$ options. Let $q$ denote the set of $q_{i}$ lists declared by the $n$ students and the $m$ colleges; $q^{t}$ is the same set when the students declare a maximum of $t$ elements. I denote by $Q$ the set of all the possible lists, with $q$ being a generic element of $Q$. Finally $Q^{t} \subseteq Q$ is the set of lists in $Q$ where each student declares a maximum of $t$ colleges and $p_{i}^{t}$ represents the list declared by a student $s_{i}$ and composed by the $t$ first colleges in $P_{i}$ in that same order. Similarly, $p^{t} \in P^{t}$.

I now define a class of mechanisms associated to the D.A.P. Each mechanism in this class, to be denoted $J(h, Q)$, results from applying the D.A.P. to the students' declaration and the preferences of the colleges. Let $h_{i}\left(q_{i} / q_{-i}\right) \in C \cup$ $\left\{s_{i}\right\}$ be the outcome of the mechanism $J$, where $h_{i}\left(q_{i} / q_{-i}\right)$ is the element that corresponds to the agent $s_{i}$ through the D.A.P. when he declares the list $q_{i}$ and the others agents declare the lists $q_{-i}$. This results in an allocation $h(q) \in \mathscr{M}$ for each $q \in Q$. If the applicants declared a maximum of $t$ colleges, for $t$ between 1 and $m$, I denote the mechanism as $J\left(h, Q^{t}\right)$. 


\section{The mechanism}

In the mechanism without limits in the number of possible declarations, it is a dominant strategy for the students to declare their preferences sincerely when S-optimal algorithm is applied (Roth [7]). The limit on the number of colleges available can affect the best strategy for students, and, with it, the allocational properties of the D.A.P. Through a simple example it can be verified that the Spanish mechanism may generate matchings that are not stable according to $P$ when the students declare sincerely their preferences.

Example 5 Consider $C=\left\{c_{1}, c_{2}\right\}$, with a maximum capacity of one position per college, and $S=\left\{s_{1}, s_{2}\right\}$, with the following preferences:

$$
P\left(c_{j}\right): s_{1}, s_{2}, c_{j} ; \quad j=1,2 . \quad P\left(s_{i}\right): c_{1}, c_{2}, s_{i} ; \quad i=1,2 .
$$

The S-optimum stable matching is $G^{*}=\left\{\left(c_{1}, s_{1}\right)\left(c_{2}, s_{2}\right)\right\}$. If the number of possible declarations is restricted to $t_{i}=1$, students declare their preferred colleges sincerely, and the previously described allocation rule is applied, the resulting new matching will be $\left\{\left(c_{1}, s_{1}\right),\left(c_{2}, c_{2}\right),\left(s_{2}, s_{2}\right)\right\}$. Given that $c_{2}$ strictly prefers to accept any of the applicants to leaving a vacant position, and $s_{2}$ prefers to be assigned to $c_{2}$ rather than remain unmatched, the pair $\left(c_{2}, s_{2}\right)$ blocks the matching, and $G^{*}$ is unstable.

None of the matchings reached through these limited mechanisms will violate the individual rationality condition. This is true because the agents' preferences allow them to individually discriminate among the elements of the other side of the market.

Hence, the matching generated by the mechanism with limits might be unstable, according to the true preferences of the agents, but it will always be individually rational. There is a limit to the distortions caused to the procedure's outcome. We can be confident that we will not meet, in equilibrium, with matchings that are not individually rational.

If a stable S-optimum mechanism without limits is applied, it is a dominant strategy for the students to declare their preferences sincerely. When a limit on the number of options exists, the mechanism can no longer guarantee this property.

Proposition 6 Given a stable S-optimum mechanism there is always a number of admissible declarations such that straightforward behavior is no longer the best strategy for any student $s_{i}$ for whom more than one college is acceptable.

Proof. Let's suppose that this statement is not true. In this case, a strategy exists that is dominant for any applicant $s_{i}$, who considers that more than one college is acceptable, irrespective of the number of colleges that he could declare $(1 \leq t<$ $m)$. Let $q_{-i}$ be the lists that are presented by the rest of the agents $\left(C \cup S /\left\{s_{i}\right\}\right)$. If $q_{i}^{*}$ is a dominant strategy for $s_{i}$ then $G_{i}\left(q_{i}^{* t} / q_{-i}^{t}\right) R_{i} G_{i}\left(q_{i}^{t} / q_{-i}^{t}\right)$ for every $q_{i}^{t}$ and every possible declaration of the other agents $\left(q_{-i}^{t}\right)$ and there exists one $q_{i}^{* t}$ for all possible $t$. In order to prove that this is not possible, it will be sufficient to 
find, for each agent $s_{i}$ one $t$ for which no strategy exists that is a better response for any $q_{-i}^{t}$.

Let's suppose that there is a $s_{i}$ such that it has $m$ elements in his preference list. It is easy to check that if the first $m-1$ colleges in $s_{i}$ 's do not declare $s_{i}$ as admissible, and $t=1$, then the best strategy for $s_{i}$ is to declare his last element as the unique option. Therefore, there are as many best strategies as $m$ colleges.

The mechanism used in the Spanish college admission system cannot guarantee that all the agents will declare their preferences sincerely. There may be limits that define games without dominant strategies for all the agents.

\section{Implementation through the J mechanism}

In this section I analyze two exercises of implementation in order to enlighten the previous results. First, I define implementation.

Let $(C, S, P, t)$ be a market and $\psi$ a concept of solution for this market. That is to say, $\psi(C, S, P, t) \subseteq \mathscr{M}$ and $\psi(C, S, P, t) \neq \emptyset$, for every market $(C, S, P, t)$. Let $(d, Q)$ be a game where $d$ is the outcome function and $Q$ the set of possible agents' declarations, and let $\phi$ be a concept of equilibrium. I say that $(d, Q)$ implements $\psi$ in a $\phi$-equilibrium if and only if, for every market $d[\phi(d, Q)]=\psi(C, S, P, t)$.

\subsection{Implementation of the stable matching set}

I will prove that the set of stable matchings when there is a limit in the number of declarations is implementable is in Nash equilibrium.

Theorem 7 The mechanism $J\left(h, Q^{t}\right)$ implements in Nash equilibrium the set $\mathscr{E}$ of stable matchings.

Proof. First I will show that every Nash equilibrium in the mechanism $J\left(h, Q^{t}\right)$ is a stable matching. Suppose it is not. I have established that the structure of agents' preferences and the mechanism guarantee that the final allocation is individually rational. So, if there is an unstable matching then a pair $\left(c_{j}, s_{i}\right)$ blocks it.

In the mechanism $J\left(h, Q^{t}\right)$ the preferences of the colleges cannot be strategically manipulated. Then, if $s_{i} P_{j} \sigma$, it exists a declaration for $s_{i}, q_{i}^{*}$, where $h_{i}\left(q_{i}^{*}, q_{-i}\right)$ is $c_{j}$. In particular, the lists $q_{i}^{*}$ could declare $c_{j}$ as a single acceptable choice. If there is a pair that blocks a stable matching, the allocation is not a Nash equilibrium.

Any Nash equilibrium can be obtained when each agent declares only the element that leads him to a particular stable matching. The result is the equilibrium and given this strategy of the remaining agents, no other agent will profit from a deviation. 
The proof of Theorem 7 only requires that the agents declare a single position. This result allows to establish the following corollary:

Corollary 8 The mechanism $J(h, Q)$ implements in Nash equilibrium the set $\mathscr{E}$ of stable matchings.

The results of Theorem 7 and Corollary 8 answer the first question: agents will not generate unstable matchings if they play Nash equilibrium.

A second question is whether or not the strategic behavior of the agents will lead them to guarantee the S-optimum stable matching.

\subsection{Implementation of the S-optimum stable matching}

In this section it will be shown how the mechanism $J\left(h, Q^{t}\right)$ implements the S-optimum matching by using Aumann's [1] definition of a strong equilibrium. This equilibrium concept assumes a high degree of collusion between the agents. A Nash equilibrium is a strong equilibrium if no coalition of players can make all its members better off by jointly deviating from the equilibrium, while taking the strategies of all other players as given.

Definition 9 A strategy $q^{t}$ is a strong equilibrium of the mechanism $J\left(h, Q^{t}\right)$ if it is a Nash equilibrium and it has the property that there is no coalition $T \subset C \cup S$ and $q^{\prime t}$ such that:

(i) $h_{k}\left(q_{-T}^{t}, q_{T}^{\prime}{ }^{t}\right) P_{k} h_{k}\left(q^{t}\right)$ for all $k \in T \cap S$, and

(ii) $h_{k}\left(q_{-T}^{t}, q_{T}^{\prime}\right) P_{k} h_{k}\left(q^{t}\right)$ for all $k \in T \cap C$.

Proposition 10 The mechanism $J\left(h, Q^{t}\right)$ implements the S-optimum stable matching in the market $(C, S, P)$ in strong equilibrium.

Proof. The fact that every strong equilibrium gives us the S-optimum matching is a direct consequence of the Theorem 4.17 in Roth and Sotomayor [9]. Their result is for the marriage model but can be directly translated to our context because the colleges has not strategic possibilities. On the other hand S-optimum stable matching can be obtained through the strategy of declaring just one element which is a strong equilibrium of the game.

\subsubsection{The non-reverse strategies}

The implementation of S-optimum stable matching in the mechanism $\mathbf{J}$ implies that, if players behave strategically according to the prescribed equilibrium, the allocative properties missed by imposing a limit on the admissible list of colleges that students can submit would be recovered. Even if straightforward behavior would lead to unstable allocations, sophisticated agents would restore stability and end up choosing the same allocation that they would have chosen when non limited. Whether this is possible or not, depends on the type of Nash equilibrium selection that is being played, and also on other possible restrictions on the set of 
admissible strategies. The same result may be achieved assuming a weaker equilibrium concept but then restricting the class of strategies that agents are assumed to be using. I identify a reasonable class, those that I call non-reverse strategies. Non-reverse strategies constitute a class of strategies closely related with the class of truncated strategies where the agents declare just the first elements of their preference list.

I require agents to restrict their declarations to those that are compatible with the information they have. In particular, their declarations must be consistent with the fact that any stable matching is necessarily a Nash equilibrium. I also demand that students do not reverse the order in which they declare colleges that may eventually correspond to them at a stable matching.

Definition 11 A strategy reverses the order of its elements when some elements of $\mathscr{E}\left(s_{i}\right)$ appear in an order different than that established in $P_{i}$, or no element of $\mathscr{E}\left(s_{i}\right)$ appears.

Theorem 12 The mechanism J $\left(h, Q^{t}\right)$ implements the S-optimum stable matching in the market $(C, S, P)$ in Nash equilibrium when the agents play non-reverse strategies.

Before proving Theorem 12, I will show two interesting results contained in Lemmas 13 and 14 . These results will be useful not only to prove Theorem 12, but also to understand the strategic context where students operate.

Lemma 13 A list $q_{i}^{t}$ whose acceptable elements are not ordered like in $P_{i}$, is dominated by another list, $g_{i}{ }^{t}$, that contains the same elements in the order determined by $P_{i}$.

Proof. I claim that $h_{i}\left(g_{i}^{t} / q_{-i}^{t}\right) R_{i} h_{i}\left(q_{i}^{t} / q_{-i}^{t}\right)$ for any $q_{-i}^{t}$. Let us suppose that the previous statement is not true. Then, there exists a set of lists $q_{-i}^{t}$ for which $h_{i}\left(q_{i}^{t} / q_{-i}^{t}\right) P_{i} h_{i}\left(g_{i}^{t} / q_{-i}^{t}\right)$. I know that $q_{i}^{t}$ and $g_{i}^{t}$ contain the same elements. The mechanism provides the student with the first college in his list that is willing to accept him once the rest of students have been placed. If $h_{i}\left(q_{i}^{t} / q_{-i}^{t}\right)$ is strictly preferred to $h_{i}\left(g_{i}^{t} / q_{-i}^{t}\right)$ then $h_{i}\left(q_{i}^{t} / q_{-i}^{t}\right) \neq h_{i}\left(g_{i}^{t} / q_{-i}^{t}\right)$, college $h_{i}\left(q_{i}^{t} / q_{-i}^{t}\right)$ has to be better than $h_{i}\left(g_{i}^{t} / q_{-i}^{t}\right)$ for $s_{i}$, and it should precede it in $q_{i}^{t}$. Then, $h_{i}\left(g_{i}^{t} / q_{-i}^{t}\right)$ is ranked above $h_{i}\left(q_{i}^{t} / q_{-i}^{t}\right)$ in $g_{i}^{t}$. This situation contradicts our hypothesis that $g_{i}^{t}$ orders its elements according to $P_{i}$.

Taking into account Lemma 13 and the fact that it is better to declare the maximum number of acceptable colleges, it can be inferred that, when a limit in the number of colleges no longer exists, declaring the truth is a dominant strategy for the students. Lemma 13 also guarantees that for any unrestricted student, to declare his preferences sincerely is a dominant strategy even if the others are restricted.

Lemma 14 When the other agents play a non-reverse strategy $\widehat{q}_{-i}$, no declaration exists where $q_{i}$ leads to a better match for agent $s_{i}$ than the one he would get in the S-optimum stable matching. 
Proof. If agents $s_{-i}$ play a strategy $\widehat{q}_{-i}$, the declarations of each one of them will satisfy two conditions. First, each agents' list include at least one college that he can achieve in stable matching on the market $(C, S, P)$. Second, the condition of non-reversion guarantees that the list declared by the agents does not change the order of the elements $e\left(s_{i}\right)$ declared. Therefore, no option exists such that an agent $s_{i}$ gets an element that is better for him than the one that he would achieve in the S-optimum stable matching.

Proof of the Theorem 12: Lemma 14 shows that playing a non-reverse strategy is a Nash equilibrium. The S-optimum stable matching may be provided in equilibrium with the mechanism $J\left(h, Q^{t}\right)$ by using non-reverse strategies. In particular, if every student plays a list where a feasible amount of $t$ stable allocations are present in order of preference from more to less then this strategy generates the required matching.

The non-reverse preference class may appear as a strong restriction over the agents' behavior. However, it does not refrain the agents for misrepresenting their preferences over the elements declared. For example, let's assume that $\mathscr{E}\left(s_{i}\right)=\left\{m_{1}, m_{2}\right\}$ with $m_{1} P\left(s_{i}\right) m_{2}$ and $s_{i}$ is allowed to declare three possible options. In the non-reverse class $s_{i}$ may declare any possible group of three colleges that includes $m_{1}$ but not $m_{2}$ or any group of three colleges that includes $m_{1}$ and $m_{2}$ ordering them in any way with $m_{1}$ preferred to $m_{2}$.

A necessary condition for a strategy $q^{t}$ that achieves the students' optimal matching in $J\left(h, Q^{t}\right)$ is that the colleges that correspond to $s_{i}$ in this matching belong to $q_{i}^{t}$ for all $i$. An additional restriction imposed in the non-reverse class concerns only the colleges on $\mathscr{E}\left(s_{i}\right)$. The reader may check how any possible strategy resulting in the students' optimal matching through $J\left(h, Q^{t}\right)$ in Nash equilibrium belongs to the non-reverse class or, as Lemma 13 shows, there exists a non-reverse strategy that dominates it.

The "natural" behavior for $s_{i}$ in $J\left(h, Q^{t}\right)$ is described by Lemma 13 . Any set of colleges declared is weakly dominated by the same set declared in the order established by $P\left(s_{i}\right)$. Each non-reverse strategy has at least one element of $\mathscr{E}\left(s_{i}\right)$ and their relative order cannot be misrepresented. Besides this fact, for all the colleges in $q_{i}^{t}$ not belonging to $\mathscr{E}\left(s_{i}\right)$, the non-reverse class describes a strategic behavior weaker that the "natural" one in the mechanism $J\left(h, Q^{t}\right)$.

The implementation result is, however, rather negative. It is necessary to impose restrictions on the set of preferences that the agents may declare. It also confirms our suspicion of strategic distortion on the declarations of the agents. This distortion forces the students not to declare the colleges they prefer, and instead declare the available ones.

\subsection{An assessment of allocational inefficiencies}

I now consider the fact that students are acting strategically by declaring preferences which do not coincide with the truth. It may happen that the announcement 
of an increase in the number of available positions in certain disciplines, generates an increase in their demand. We may be facing a process where the supply creates its own demand. In this case, if the lists of the students are considered as a social demand of higher education the problem becomes critical.

Example 15 Let market $(C, S, P)$ be composed of two sets of agents: $\left(s_{1}, s_{2}, s_{3}\right)$ and $\left(c_{1}, c_{2}\right)$, where the colleges have two vacancies in $c_{2}$ and one in $c_{1}$. The agent's preferences are the following:

$P\left(s_{i}\right): c_{1}, c_{2}, s_{i}$; for all $i=1,2,3 ; \quad P\left(c_{1}\right): s_{1}, s_{2}, s_{3}, c_{1} ; \quad P\left(c_{2}\right): s_{1}, s_{2}, s_{3}, c_{2}$.

It is easy to check that there is only one stable matching, $\left\{\left(c_{1}, s_{1}\right),\left(c_{2}, s_{2}, s_{3}\right)\right\}$. Additional limitations could be established by allowing the students to declare one option only. The process can be made dynamic by establishing a rule for the creation of new colleges. The procedure will be the following: at each new period, a new student appears with the same preferences as the existing ones. He will be able to declare one college. Alongside the supply we create, every period, a new position in the college most demanded in the previous one.

It is easy to verify that the declarations of the students in period 1 compatible with the stable matching are: $P\left(s_{1}\right)=c_{1}, P\left(s_{2}\right)=c_{2}$, and $P\left(s_{3}\right)=c_{2}$. Therefore in period 2 college $c_{2}$ will have a quota of three and the only stable matching of the market will be $\left\{\left(c_{1}, s_{1}\right),\left(c_{2}, s_{2}, s_{3}, s_{4}\right)\right\}$. Where $s_{4}$ is our new student who will have declared $c_{2}$,since this is his only option compatible with a stable matching when $t=1$. This situation forces the growth of college $c_{2}$ even when the students unanimously prefer college $c_{1}$.

The distortion generated worsens its allocational effects as time goes by. However, in Spain, every year, most of the students end up in colleges declared as their first option. This situation is interpreted by the administration as evidence that the process works properly. Given the previous results, further study needs to be done.

To verify the real amount of the distortion, two simple tests may be run. Both tests are based on making a portion of the student population reveal their preferences sincerely. For that, it is enough to apply the D.A.P. without restrictions. Lemma 13 proves that any student able to declare his preferences without limitations should do it sincerely. We may select a group of students and allow them to declare their preferences. The statistical comparison of the frequencies in which the students apply for the different colleges in the restricted and unrestricted samples should give a clear idea of the distortion induced by the strategic behavior of the agents. Another possibility is to compare the frequencies of the students' first options. In that case the test will reveal possible allocative problems.

If the test does not show any significant differences between the two samples we do not need to worry; otherwise, the possibility of eliminating the limitations in the students' declarations should be seriously considered. If for any reason the administration prefers to maintain a limitation, a group of students can be profitably maintained as an unrestricted sample for control purpose. This group would allow us to establish what is the generated distortion and would provide 
us with trustworthy information about the preferences of the agents. This information may also be used to set down a policy for further supply of new positions in higher education.

\section{Conclusions}

I have pointed out how an apparently innocuous alteration of the D.A.P. implies some meaningful consequences. Limiting the number of options that students can declare leads to a perverse action that hinders stable matching. It also strengthens the strategic behavior of the agents. The mechanism finally fulfills its allocative goals. Unfortunately these properties are recovered at the expense of a pattern of behavior which is not straightforward. For this reason, the quality of the information provided by the mechanism and the level of satisfaction of the students involved needs to be verified.

To eliminate the limitation imposed over the students' declarations appears as a fundamental conclusion of the paper. Otherwise, the intention to satisfy the students' demand could lead us to an extreme case: the creation of new positions in the most solicited colleges instead of those socially more desired.

\section{Endnote}

${ }^{1}$ We assume that no agent is indifferent between any two potential mates, or between any possible mate and the unmatched option.

\section{References}

[1] Aumann, R.J. (1959) Acceptable Points in General Cooperation n-Persons Games. In: Contributions to the Theory of Games IV. Princeton University Press, Princeton

[2] Escudero, T. (1991) Acceso a la Universidad: Modelos Europeos, Vías Alternativas y Reformas en España. INFORMES 36, Instituto de Ciencias de la Educación, Universidad de Zaragoza

[3] Gale, D., Shapley, Ll. (1962) College Admissions and Stability of Marriage. Amer. Math Monthly 69: 9-15

[4] Gusfield, D., Irving, R.W. (1989) The Stable Marriage Problem, Structure and Algorithms. The M.I.T. Press, Cambridge, Massachusetts

[5] Roth, A.E. (1984) Stability and Polarization of Interests in Job Matching. Econometrica 52: $47-57$

[6] Roth, A.E. (1984) Misrepresentation and Stability in the Marriage Problem. J. Econ. Theory 34: 383-387

[7] Roth, A.E. (1985) The college Admissions Problem is not Equivalent to the Marriage Problem J. Econ. Theory 36: 277-288

[8] Roth, A.E., Sotomayor, M. (1989) The College Admissions Problem Revised. Econometrica 57: $559-570$

[9] Roth, A.E., Sotomayor, M. (1990) Two-Sided Matching Markets: A Study in Game-Theoretic Modeling and Analysis. Econometric Society Monograph. Cambridge University Press, Cambridge 